\title{
ANÁLISE DA RECUPERAÇÃO DA INFORMAÇÃO EM CATÁLOGO ONLINE DE BIBLIOTECA UNIVERSITÁRIA
}

\author{
ANÁLISIS DE LA RECUPERACIÓN DE LA INFORMACIÓN EN \\ CATÁLOGO EN LÍNEA DE BIBLIOTECA UNIVERSITARIA
}

\begin{abstract}
Susana Yuri Okada syuok@usp.br Graduada em Ciências da Informação e da Documentação pela Faculdade de Filosofia, Ciências e Letras da Universidade de São Paulo / Campus de Ribeirão Preto. Técnica de documentação e informação da Biblioteca Central da Universidade de São Paulo / Campus de Ribeirão Preto
\end{abstract}

Cristina Dotta Ortega ortega@eci.ufmg.br Graduada em Biblioteconomia e Documentação. Mestre e Doutora em Ciência da Informação pela Escola de Comunicações e Artes da Universidade de São Paulo. Professora da Escola de Ciência da Informação da UFMG.

\begin{abstract}
Resumo
Objetiva analisar a recuperação da informação em catálogo on-line de biblioteca universitária, por meio da observação da entrada de dados realizada em formato MARC 21. A metodologia inclui revisão bibliográfica que conceitua e caracteriza regras de catalogação e formatos de registro bibliográfico, apresentando o papel de ambos e de outras variáveis nos resultados da recuperação da informação. Inclui também análise dos resultados de duas buscas realizadas no catálogo geral de bibliotecas da Universidade de São Paulo. Esta análise demonstra que, uma tarefa que parece ser simples como a busca em catálogos, pode apresentar problemas aos usuários. Conclui que os bibliotecários e auxiliares envolvidos na prática da catalogação devem possuir domínio sobre os processos e instrumentos de tratamento da informação da unidade de trabalho em que atuam, domínio esse que implica em capacidade investigativa, interpretativa e de tomada de decisão.
\end{abstract}

\section{Palavras-chave}

Catálogo on-line; Bibliotecas universitárias; Recuperação da informação; Formato MARC; AACR2

\section{INTRODUÇÃO}

O catálogo de uma biblioteca universitária é uma rica fonte de informação. Saber tratar a informação para compor o catálogo é de suma importância para que se tenha uma recuperação rápida e eficaz. 
O registro de um catálogo é uma estrutura para representação de um documento. Esta estrutura pode ser obtida por meio de um formato de registro bibliográfico e de regras de catalogação. As regras de catalogação são concebidas a partir de certos princípios que objetivam a identificação de um documento de forma unívoca. $\mathrm{O}$ tratamento de um livro, por exemplo, vai além do cadastramento no sistema para controle do acervo.

Conhecer e utilizar um formato e regras de catalogação é um dos desafios do profissional responsável pela alimentação de um catálogo de uma biblioteca. A recuperação de informações será o reflexo do seu trabalho e, ao mesmo tempo, objeto de estudo para melhorar o tratamento. Essa recuperação da informação no catálogo, que é o processo final de várias etapas de trato com a informação, parece ser o típico problema a ser resolvido pelo setor de referência, o qual possui especialistas que auxiliam o usuário na busca de informações pertinentes à sua pesquisa. No entanto, não há habilidade de busca que supere modos inconsistentes de organização da informação.

O estudo foca a forma como se alimentam catálogos on-line de bibliotecas universitárias que adotam formatos de registro bibliográfico (neste caso, o formato MARC 21 Machine Readable Cataloguing). Aborda-se apenas o aspecto da descrição formal do documento, ou seja, a representação descritiva (e não a representação do conteúdo), analisando e, principalmente, buscando compreender como os princípios em que se baseiam as regras são adotados, a fim de verificar sua influência sobre a recuperação da informação.

\section{A BIBLIOTECA UNIVERSITÁRIA}

A universidade procura manter a qualidade de seu ensino e formar pessoas nas mais diversas áreas do conhecimento. Visa a preparar profissionais atentos a acompanhar transformações, sociais, econômicas e culturais. A atenção para a investigação e para o conhecimento é despertada e faz com que o desejo de formação e transformação seja projetado para além da vida acadêmica.

As reflexões sobre o ensino superior passam pela biblioteca universitária. Uma biblioteca universitária é voltada a atividades de ensino, pesquisa e extensão, para o que, promove a recuperação, disseminação e uso de informações que subsidiem estas atividades.

Segundo a American Library Association (ALA), a biblioteca universitária é definida "como una biblioteca o sistema de éstas, establecida, mantenida y administrada por una 
universidad para cubrir las necesidades de información de sus estudiantes y apoyar sus programas educativos, de investigación y demás servicios" (apud LÓPEZ YEPES, 2004, v. 1, p. 209).

A biblioteca universitária não é estática, pois deve observar como se dá a informação no mundo contemporâneo e participar do processo de crescimento e de mudança das demandas institucionais por informação.

\section{O CATÁlogo e A CATALOGAÇÃo}

O catálogo é um dos instrumentos mais antigos de descrição de uma coleção de documentos em uma biblioteca e está sempre vinculado a um acervo de uma instituição (LÓPEZ YEPES, 2004; MEY, 1995b). Através dele, o usuário tem acesso a este acervo. Se até o início do período moderno o catálogo era visto apenas como o inventário da coleção, nos dias atuais, deve possibilitar a identificação e localização de seus documentos com rapidez e facilidade, e informar os conteúdos (assuntos) a eles atribuídos.

O catálogo deve apresentar:

- registros uniformes;

- $\quad$ informação precisa e atual;

- economia de recursos e de tempo para produção e gestão; e

- fácil acesso e consulta (LÓPEZ YEPES, 2004; MEY, 1995b).

Essas características permitem o aprimoramento desse canal de comunicação entre documentos e usuários que é o catálogo da biblioteca.

A catalogação pode ser definida como o processo de descrição dos elementos informativos que permitem identificar um documento e de indicação dos pontos de acesso que remetam a estes elementos. Os pontos de acesso devem ser controlados de modo a evitar ambiguidade ou dúvida na informação, sendo escolhidos e determinados pelo profissional, de acordo com normas, como por exemplo, o Anglo-American Cataloguing Rules (AACR2). Nos catálogos de fichas em papel, produzidos segundo o AACR2, os pontos de acesso são a(s) responsabilidade(s) pelo conteúdo intelectual, título, série e assunto. Nos catálogos automatizados, os pontos de acesso são muitas vezes ampliados, já que a tecnologia eletrônica opera de modo mais rápido e com maior quantidade de dados. Em ambos os casos, deve-se seguir critérios para o preenchimento dos campos recuperáveis. 
A utilização de regras de um código normalizado para padronização visa organizar e facilitar o acesso a um documento, evitando uma catalogação duplicada e facilitando o intercâmbio do registro (SVENONIUS, 1989).

Existem diversos códigos de catalogação nacionais como as regras de catalogação portuguesas, as Reglen für die Aphabetische Katalogisierung (RAK) alemãs, as COST 7.1 soviéticas, as espanholas, as francesas, as italianas, entre outras (RÍOS HILARIO, 2003). Dentre as normas internacionais para representação descritiva, pode-se citar a International Standard Bibliographic Description (ISBD) e o AACR2².

A ISBD representa um caminho para a padronização, mas não contempla os requisitos sobre os pontos de acesso, tratando apenas das áreas da descrição bibliográfica (MEY, 1995b). O AACR2, embora baseado na ISBD quanto ao sistema de pontuação, áreas e elementos essenciais e ordem das mesmas, e possuir um capítulo (capítulo 21) específico para a escolha dos pontos de acesso, item importante para a recuperação da informação, não é um instrumento de trabalho de fácil manuseio e compreensão (BARBOSA, 1978).

O AACR, assim como a maioria dos códigos, foi baseado nos Princípios de Catalogação acordados em Paris em 1961 (os chamados Princípios de Paris) (BARBOSA, 1978). Ainda que a automação da criação e gestão dos catálogos das bibliotecas não estivesse em pleno funcionamento na época da publicação do AACR (1967), o assunto não foi ignorado. Em sua segunda edição, o AACR2 (1978), foi incorporada a descrição para diversos tipos de materiais (além do livro) e as orientações da ISBD, como a pontuação, necessária para a automação (BARBOSA, 1978; GARRIDO ARILLA, 1996), segundo se entendia então.

Apesar das atualizações do AACR2 (a última é de 2002 e recebeu a sigla AACR2R), a sua redação continua complexa e pode ser um ponto a exercer implicações na interpretação do código. Além disso, utilizar um código elaborado para catálogos em suporte papel (em geral, em fichas) pode não contemplar as necessidades da catalogação atual, influenciando o tratamento da informação e, por consequência, a sua recuperação.

No Brasil, após várias discussões, o AACR2 foi recomendado e adotado como código de catalogação nacional, com tradução e distribuição realizada pela Federação Brasileira de Associações de Bibliotecários, Cientistas da Informação e Instituições (FEBAB).

${ }^{2}$ O AACR2 é de fato um código anglo-americano, mas acabou ganhando dimensão praticamente internacional. 
Além de servir como orientação para a catalogação, um código nacional é um dos elementos necessários para a viabilização de projetos de catalogação cooperativa, os quais trazem benefícios em termos de tempo e custo.

$\mathrm{Na}$ catalogação cooperativa, a catalogação de um documento é feita apenas uma vez e o registro resultante é disponibilizado a outras bibliotecas por meio de um catálogo coletivo, ou seja, existe um registro original que é compartilhado por um grupo de unidades de informação, agilizando o serviço.

Para que um registro seja mais bem reaproveitado no meio eletrônico, é necessário que se adote formato e código de catalogação que permita a importação sem grandes custos de conversão de dados e/ou de revisão posterior dos mesmos.

Essa atividade cooperativa pode se dar em nível institucional, local, regional, nacional e internacional (LÓPEZ YEPES, 2004). No nível internacional, tem-se o exemplo do Online Computer Library Center (OCLC).

No Brasil, o exemplo mais significativo de catalogação cooperativa é o da Rede Bibliodata, cujos trabalhos tiveram início em 1980 objetivando a produção de um catálogo coletivo nacional pelas bibliotecas brasileiras que também subsidiasse seu próprio trabaIho (HÜBNER, 2002).

\section{FORMATOS DE REGISTROS BIBLIOGRÁFICOS}

Formato de registro bibliográfico é "um padrão para o tratamento informatizado do dado bibliográfico" (ORTEGA, 2002, p. 75).

Um formato tem como função,

permitir que qualquer elemento significante de um documento possa ser identificado com precisão por meio de processo automatizado, possibilitando por conseguinte a seleção, manipulação e recuperação do registro ou elemento de dado (LINFORD, 1987 apud LOBO, 1990, p. 15).

Martin (1997), por sua vez, ao definir um formato, trabalha com a ideia de dados que são organizados para representar uma classe de objetos. Essa representação se dá de modo informatizado e os dados são conceituados através de normas, como a ISBD e o AACR2.

A literatura sobre formatos de registro bibliográfico está, em geral, centrada nas características do formato MARC, como segue. 
Pode-se citar os seguintes elementos constituintes dos formatos, em termos de estrutura para os registros:

- campos: cada área específica dentro de um registro;

- $\quad$ subcampos: as partes dos campos; e

- indicadores: códigos associados a cada campo que fornecem informações sobre o conteúdo ou forma do campo ou ainda sobre o tipo de ação desejada em certos processos de manipulação de dados, tais como a alfabetação (FURRIE, 2000; GARRIDO ARILLA, 1996; MELGAÇO, 1987) ${ }^{3}$.

Quanto aos tipos de campos, os formatos podem apresentar:

- $\quad$ campos de dados de controle variável: em geral, são campos de extensão fixa. Não possuem indicadores ou subcampos e não são repetitivos; são preenchidos por códigos e não por informação textual. Foram criados com o objetivo de possibilitar a busca, uma vez que muitos campos se baseiam em tabelas de códigos ou formas sucintas padronizadas (BYRNE, 2001) ${ }^{4}$.

- $\quad$ campos de dados variáveis: são campos de tamanho variável, podendo ser repetitivos ou não, dependendo da natureza da informação; são preenchidos por informação textual, e estão presentes em todos os formatos de registros bibliográficos (ORTIZ-REPISO JIMÉNEZ, 2006).

A implantação de um formato implica em estruturas com funções próprias, quais sejam:

- formato de entrada de dados: formulário de captação de dados, ou seja, formulário visto pelo alimentador do catálogo, a partir do qual os dados são registrados e armazenados em arquivos;

- $\quad$ formato do arquivo de armazenamento dos dados: estrutura do arquivo gerado por meio da entrada de dados. A partir do arquivo de armazenamento interno, é gerado $o$ arquivo para intercâmbio de dados e os formatos de apresentação;

\footnotetext{
${ }^{3}$ Alguns formatos como LILACS e CEPAL não fazem uso de indicadores (BIREME, 2008) e (ARENDT, 2003).

${ }^{4}$ Os campos de dados de controle variável são evidentes na entrada de dados de sistemas que adotam o formato MARC.
} 
- $\quad$ formato de intercâmbio: formato padronizado, ou seja, padrão aceito internacionalmente para que haja o intercâmbio de registros entre sistemas computadorizados de uma forma econômica e eficiente; e

- $\quad$ formato de apresentação: é a forma de apresentação dos dados ao usuário final ou intermediário, seja ela impressa ou eletrônica (BALBY, 1995; LOBO, 1990; MELGAÇO, 1987).

Os formatos de intercâmbio e de apresentação são, de fato, formatos de saída. O formato de intercâmbio também é denominado formato de comunicação (CYSNEIROS, 1987).

Para que a função de intercâmbio do formato possa ocorrer, ou seja, para que haja a exportação de dados estruturados no meio informatizado de um sistema com aproveitamento por outro, são necessários os seguintes componentes:

- a convenção dos campos de informação do registro: por exemplo, no caso do formato MARC, o campo 100 será destinado ao autor principal e o campo 245, ao título. Este é o formato propriamente dito: os campos para o tratamento da informação;

- $\quad$ a convenção para a entrada das informações, ou seja, a forma de preencher os campos, segundo regras de catalogação (como AACR2 e ISBD), notações de classificação bibliográfica (como CDD e CDU) e vocabulários controlados para indexação. Por exemplo: para se estabelecer a forma de entrada do sobrenome do autor, pode-se utilizar as regras de catalogação, como as do AACR2; e

- o lay-out físico dos registros no meio magnético: a estrutura necessária para gerar o arquivo de intercâmbio, como aquela indicada pela norma ISO 2709, a qual deve estar previamente implementada no software adotado (MARCONDES; SAYÃO, 1991).

A norma ISO 2709 estabelece a forma de intercâmbio entre os sistemas por meio da estruturação dos dados no meio físico de armazenamento e é um padrão aceito internacionalmente. $O$ arquivo de intercâmbio gerado por esta norma possui a seguinte estrutura:

- líder: campo codificado de tamanho fixo, considerado a etiqueta do registro, que apresenta informações como tipo e situação do registro;

- diretório: série de entradas numéricas contendo a tag (etiqueta do campo), a posição inicial e o tamanho de cada campo; 
- $\quad$ conteúdos propriamente ditos dos campos de dados bibliográficos como autor, título, etc.; e

- $\quad$ separadores de campo e de registros (pontuação) (FERREIRA, 2002; LAFUENTE LÓPEZ ; GARDUÑO VERA, 2001; ROBREDO, 2005).

Atualmente, o intercâmbio de registros pode ser realizado com uso da linguagem de marcação EXtensible Markup Language (XML), a qual permite que sejam transportados de um sistema a outro tanto a estrutura quanto o conteúdo dos registros.

A escolha e uso de um formato permite agilidade e qualidade por possibilitar processos como: conversão retrospectiva (conversão de fichas catalográficas em papel para registros bibliográficos em meio eletrônico, com o uso de normas e padrões estabelecidos) e catalogação por cópia (importação de registros bibliográficos de outros repositórios, em geral, em processos de catalogação cooperativa).

O software utilizado é variável importante, pois os formatos apresentam características que precisam ser viabilizadas tecnologicamente, e esta escolha exerce influência nos processos de recuperação da informação, assim como nos processos de intercâmbio de registros.

Os formatos de registro bibliográfico definem-se em parte pela incorporação de recursos tecnológicos, e seu uso relaciona-se à catalogação cooperativa ou às redes de informação especializada. Quanto a estas características, observamos variação terminológica significativa, sob a predominância do termo 'formatos de intercâmbio':

É recorrente, na literatura latino-americana (exceto a do Brasil), a adoção dos termos 'formato de registro de informação bibliográfica' e 'formato de registro bibliográfico' e não 'formato de intercâmbio'. (...) O termo 'formato de registro bibliográfico' é mais abrangente, uma vez que não restringe as funções de um formato unicamente ao intercâmbio de dados. Também evita a indistinção entre formatos de registro bibliográfico propriamente ditos, como o formato MARC e o formato IBICT, e um dos seus componentes, ou seja, a norma estabelecida para intercâmbio de dados bibliográficos em meio magnético, como a ISO 2709 (ORTEGA, 2002, p. 76).

Ou seja, o formato de registro bibliográfico propriamente dito (convenção dos campos de informação do registro) é muitas vezes tomado pela sua função de intercâmbio, como demonstram definições recorrentes sobre formato pautadas nos elementos que compõem a estrutura do arquivo de intercâmbio (líder, diretório, conteúdos, e separadores de registros e de campos).

O termo formato de registro relaciona-se ainda a outros usos, como formatos de entrada, de armazenamento e de apresentação, segundo tratado acima. 
A distinção entre termos, neste caso, é necessária para explicitar os conceitos que permitem o entendimento de operações concretas.

\subsection{Formato Marc}

Pode-se dizer que o projeto MARC teve origem em relatório publicado em 1963 sobre a informatização da Library of Congress (LC) dos Estados Unidos. Entre 1966 e 1968, a LC testou o formato MARC em 16 bibliotecas do país. Apenas posteriormente o formato foi adaptado ao AACR (GARRIDO ARILLA, 1996).

Entre 1982 e 1987, o formato MARC consolidou-se em duas aplicações básicas de bibliotecas: como formato de intercâmbio de informação bibliográfica e como formato orientador para o desenho de bases de dados bibliográficas (LAFUENTE LÓPEZ; GARDUÑO VERA, 2001).

Variações do formato MARC foram surgindo com o tempo, como por exemplo, USMARC (Estados Unidos), UKMARC (Inglaterra), INTERMARC (França), IBERMARC (Espanha), CANMARC (Canadá), entre outros. Visando maior uniformidade e compatibilidade, as instituições adotaram o formato dominante USMARC e, posteriormente, o MARC 21, desenvolvido pela fusão entre o USMARC e o CANMARC, cujo manual foi publicado em 1999 (GARRIDO ARILLA, 1996; LAFUENTE LÓPEZ; GARDUÑO VERA, 2001).

O formato UNIMARC foi criado em 1977 pela IFLA, voltado à descrição de monografias e publicações seriadas, como um formato de intercâmbio entre agências nacionais de catalogação; cada instituição trabalha em seu formato local ou nacional, segundo as regras de catalogação de cada país, mas emite registros UNIMARC (GARRIDO ARILLA, 1996). Apesar de ter sido criado como formato de intercâmbio, o formato UNIMARC é mais utilizado como formato de registro do que em sua função de intercâmbio, constituindo-se, junto com o MARC 21, como as maiores fontes de definição para uso deste formato (CORDEIRO, 2008).

No Brasil, em 1972, Alice Príncipe Barbosa adaptou o formato MARC, dando-lhe o nome de Catalogação Legível por Computador (CALCO) e propondo seu uso no país. Foi com esse formato que a Fundação Getúlio Vargas, no Rio de Janeiro, criou a então rede Bibliodata/CALCO de catalogação cooperativa, que atualmente faz uso do formato MARC 21. O formato IBICT (Formato de Intercâmbio Bibliográfico e Catalográfico), baseado no CALCO, foi editado em 1987 pela instituição que lhe deu o nome, como proposta de for- 
mato-padrão para o país (ORTEGA, 2002). O formato MARC é cada vez mais adotado nas bibliotecas brasileiras.

O formato MARC é constituído por cinco tipos de formatos coordenados, os quais compõem os chamados formatos MARC 21:

- $\quad$ Formato para dados bibliográficos: contém especificações para codificar os elementos de dados ${ }^{5}$ necessários para descrever, recuperar e controlar várias formas de material bibliográfico (um livro, uma publicação seriada, um registro sonoro, mapas, entre outros documentos) (LIBRARY OF CONGRESS, 1996).

- $\quad$ Formato para dados de autoridades ${ }^{6}$ : permite criar registros contendo formas padronizadas para pessoas, entidades coletivas, eventos, títulos e assuntos; permite ainda estabelecer uma forma reconhecida para um nome e usar esta forma como ponto de acesso em um registro (LIBRARY OF CONGRESS, 2004).

- $\quad$ Formato para dados de coleção (holdings): contém especificações para codificar elementos de dados pertinentes aos dados de coleções e a localização de todos os tipos de materiais (LIBRARY OF CONGRESS, 1996).

- $\quad$ Formato para dados de classificação: contém especificações para codificar elementos de dados relacionados aos números de classificação e aos dados associados a eles; são usados para a manutenção e desenvolvimento de esquemas de classificação (LIBRARY OF CONGRESS, 1996).

- $\quad$ Formato para informação comunitária: fornece especificações para registros que contêm informações sobre eventos, programas, serviços etc., que podem ser integradas ao catálogo de acesso público (LIBRARY OF CONGRESS, 1996).

O formato bibliográfico MARC 21 é mais complexo, diferenciando-se de outros por conter maior nível de detalhamento em sua estrutura, como no caso dos indicadores, dos campos de dados de controle variável e dos pontos de acesso principal e secundários. Anteriormente as fichas do catálogo para um mesmo documento eram chamadas de entradas (principal e secundárias), já que funcionavam como pontos de acesso distintos. Winke (1993, p. 58) trata a questão do seguinte modo:

um catálogo on-line tem apenas uma entrada (ou seja, um registro) para cada publicação existente na coleção, e não se justifica que ela seja de-

\footnotetext{
${ }^{5}$ Elementos de dados são os conteúdos transcritos para cada campo.

${ }^{6}$ Formatos de dados de autoridades, ou formatos de nomes autorizados, segundo tradução mais condizente com a língua portuguesa, como comenta Mey (1995a).
} 
nominada entrada principal, pois não existem outras entradas para esta publicação específica (ou seja, entradas secundárias que seriam, na realidade, registros duplicados).

Se o usuário realizar uma busca pelo nome de autor, independente de o mesmo receber a denominação de entrada principal (campo 100 do formato MARC 21) ou secundária (campo 700), o nome constará no índice de busca, pois o sistema deve ser programado para tal. Ao profissional, cabe selecionar o campo que corresponde à função da autoria e padronizar a forma de entrada para que a recuperação seja possível ${ }^{7}$.

O formato MARC 21 é utilizado em atividades de intercâmbio de registros bibliográficos em escala mundial. Atualmente foi ampliado para atender ao tratamento e localização de documentos eletrônicos.

Segue análise de registros MARC, criados a partir de uma destas implementações.

\section{ANÁLISE DE REGISTROS}

Foram analisados dois registros, pesquisados no banco de dados bibliográficos da Universidade de São Paulo (USP), o Dedalus, em dezembro de 2008. O Dedalus ${ }^{8}$ é "o instrumento oficial incumbido de reunir todos os registros relativos à informação bibliográfica, promover a sua recuperação e indicar a localização física do material no acervo das bibliotecas da USP" (UNIVERSIDADE DE SÃO PAULO, 2009, p.1). É gerenciado pelo Sistema Integrado de Bibliotecas (SIBi) que administra uma rede de 42 bibliotecas, onde cada biblioteca é responsável pela alimentação de seu catálogo. Através do catálogo geral é possível fazer a busca dos materiais existentes no acervo (livros, teses, produção científica do corpo docente, etc.) de todas as bibliotecas participantes (UNIVERSIDADE DE SÃO PAULO, 2001). Em 1997 o Dedalus foi “implementado com o software Aleph 300 (Automated Library Expandable Program), desenvolvido na The Hebrew University, em Jerusalém, Israel e comercializado pela empresa Ex Libris" (NOGUEIRA; SALEM, 2001, p.1). O Dedalus utiliza o formato MARC 21 para entrada de dados.

Os registros analisados apresentam inconsistências na sua elaboração, uma vez que o mesmo documento foi descrito de formas diferentes no catálogo. Não houve intenção de criticar a política estabelecida pela instituição ou os procedimentos de trabalho realizados por seus profissionais, apenas de levantar e discutir problemas que ocasionam dificuldades de recuperação.

\footnotetext{
${ }^{7}$ Cabe lembrar que a determinação da entrada principal, no sentido de ponto de acesso principal do registro, exerce função relevante na ordenação de listas de referências.

${ }^{8}$ Disponível em: <http://dedalus.usp.br:4500/ALEPH/por/USP/USP/DEDALUS/START>.
} 
Utilizou-se o catálogo disponibilizado pela USP na atualidade, mas não se pode deixar de considerar que muitos registros foram gerados antes da união dos catálogos de cada biblioteca em um único catálogo geral, podendo constar ainda peculiaridades destes catálogos locais. Muitos desses registros também podem ter sido criados por meio do software anteriormente utilizado e importados para o software atual Aleph apresentando ainda hoje características intrínsecas ao sistema antigo ou decorrentes de dificuldades geradas por seus processos de alimentação.

Dentre os recursos de busca disponíveis no catálogo Dedalus, adotou-se a opção denominada Índice e foram selecionadas uma busca feita por título e outra por autor, por serem as formas mais comuns de busca efetuadas pelos usuários. O Índice é recurso de busca que apresenta o conteúdo presente dos campos. A análise em questão trata apenas de registros referentes a livros, portanto, está baseada nas regras de catalogação unicamente para este tipo de documento.

\subsection{Busca por Autor}

Efetuou-se a busca pelo nome Antonio Miranda, referindo-se ao professor do Departamento de Ciência da Informação da Universidade de Brasília (UnB).

A busca apresentou os seguintes resultados:

\begin{tabular}{|l|l|}
\hline 14 & Miranda, Antonio \\
\hline 16 & Miranda, Antonio 1940- \\
\hline 1 & Miranda, Antônio A. de \\
\hline 1 & Miranda, Antonio Batista de \\
\hline 1 & Miranda, Antonio Carlos de \\
\hline 3 & Miranda, Antonio dos Passos \\
\hline 1 & Miranda, Antonio Jose Bernardes \\
\hline 1 & Miranda, Antonio L C de \\
\hline 3 & Miranda, Antonio Lisboa Carvalho de \\
\hline 1 & Miranda, Antônio Lisboa Carvalho de \\
\hline
\end{tabular}

Quadro 1: Resultado da busca pelo Índice Autor, Base Livros, Inicia índice em: Miranda, Antonio (Resultado total).

Fonte: Universidade de São Paulo - Dedalus (2008)

Nota-se a grande variedade de resultados referentes aos pontos de acesso de nome de autor para Miranda, Antonio.

Se há várias formas para o mesmo nome do autor, o usuário deve ter o cuidado de executar a busca por cada uma delas, o que exige mais tempo e memória do usuário para 
retornar aos registros do mesmo nome de autor. O catálogo analisado possui remissivas de autores, mas elas não foram implementadas no registro analisado.

No caso da busca pelo nome de autor Antonio Miranda, embora vários registros tenham sido recuperados (14 registros), o usuário deixará de recuperar documentos que possam interessar, caso desconheça o nome completo deste autor (Antonio Lisboa Carvalho de Miranda - 3 registros; Antônio Lisboa Carvalho de Miranda - 1 registro; Antonio L C de Miranda - 1 registro), ou não venha a verificar os registros cujo ponto de acesso de nome do autor inclua data relacionada ao nome (16 registros).

A duplicação de nome de autor pode gerar dúvidas ao usuário, por encontrar nomes parecidos que se diferenciam por alguns detalhes, como os apresentados, levando até a que não localize registros de documentos de interesse.

\subsection{Busca por Título}

O título utilizado para a busca foi "Para entender as linguagens documentárias". Obteve-se o seguinte resultado:

\begin{tabular}{|c|c|l|l|}
\hline 1 & 0555913 & $\begin{array}{l}\text { Para entender as linguagens do- } \\
\text { cumentárias }\end{array}$ & 1994 \\
\hline 2 & 1261805 & $\begin{array}{l}\text { Para entender as linguagens do- } \\
\text { cumentárias }\end{array}$ & 2002 \\
\hline 3 & 1451902 & $\begin{array}{l}\text { Para entender as linguagens do- } \\
\text { cumentárias }\end{array}$ & 2002 \\
\hline 4 & 1533546 & $\begin{array}{l}\text { Para entender as linguagens do- } \\
\text { cumentárias }\end{array}$ & 2005 \\
\hline
\end{tabular}

Quadro 2: Resultado da busca pelo Índice Título, Base Livros, Inicia índice em: Para entender as linguagens.

Fonte: Universidade de São Paulo - Dedalus (2008)

Analisando-se os registros duplicados (2 e 3), tem-se:

Título Para entender as linguagens documentárias / Anna Maria Marques Cintra ... [et al.]

Imprenta São Paulo, SP : Polis, 2002

Descr Fís $92 \mathrm{p}$

Série Palavra-chave : 4

Autor Séc Cintra, Anna Maria Marques;

ISBN 8572280012X

Quadro 3: Registro duplicado do livro de título "Para entender as linguagens documentárias" (Registro 2).

Fonte: Universidade de São Paulo - Dedalus (2008)

Título Para entender as linguagens documentárias

Edição 2. ed. Rev ampl 


\begin{tabular}{|c|c|}
\hline Imprenta & São Paulo : Polis, 2002 \\
\hline $\begin{array}{l}\text { Descr } \\
\text { Fís }\end{array}$ & $92 p$ \\
\hline Série & Coleção Palavra-Chave ; 4 \\
\hline $\begin{array}{l}\text { Autor } \\
\text { Séc }\end{array}$ & $\begin{array}{l}\text { Cintra, Anna Maria Marques; Tálamo, Maria de Fátima Gonçalves Moreira; Lara, Maril- } \\
\text { da Lopes Ginez de; Kobashi, Nair Yumiko; }\end{array}$ \\
\hline$I S B N$ & $857228012 X$ \\
\hline
\end{tabular}

Quadro 4: Registro duplicado do livro de título "Para entender as linguagens documentárias" (Registro 3).

Fonte: Universidade de São Paulo - Dedalus (2008)

O profissional, com o auxílio do AACR2 e com base na política institucional, é quem determina quais informações devem ser consideradas no momento do tratamento da informação. Nota-se, na figura 3 e 4, apesar dos dois registros indicarem o ano de 2002, que foi adotado tratamento distinto para a descrição e pontos de acesso no que se refere à responsabilidade compartilhada. Na figura 4, a despeito das regras do AACR2, optou-se pela indicação dos quatro nomes de autores como pontos de acesso e não apenas do primeiro autor (quando se têm mais de três autores), além de se omitir a indicação de responsabilidade no campo título, optando-se por colocar essa informação, somente no ponto de acesso referente à autoria secundária.

Outra informação que também gera dúvidas é a da edição do livro do ano de 2002, pois a mesma consta apenas no registro da figura 4 . No registro da figura 3 , subentendese que este livro de 2002 seja primeira edição.

Nota-se também a ausência de padronização quanto ao registro do nome da série, uma vez que a palavra Coleção só é inserida se estiver presente no nome da série na fonte principal de informação do documento.

No caso do título analisado houve duplicação de registros. Os registros duplicados tomam o tempo do usuário e o confundem nas tarefas de identificação do documento e de localização do mesmo em bibliotecas da rede. Há de fato um grande risco de o usuário não efetivar devidamente estas tarefas.

\section{CONCLUSÕES}

Após a análise efetuada, mesmo que em uma pequena amostra, verifica-se que pode haver problemas na alimentação de um catálogo on-line de biblioteca universitária. Rico em detalhamento, percebe-se que a forma de alimentar um catálogo baseado no 
formato MARC 21 acarreta problemas na recuperação do registro e identificação do documento.

Recupera-se facilmente a informação quando os registros dos mesmos documentos são únicos, não induzindo dúvidas quanto à autoria ou título dos mesmos. Como foi observado, muitas vezes os registros duplicados para o mesmo documento podem confundir o usuário, assim como a duplicação de nome de autor.

A busca em uma base de nomes autorizados, renomada e/ou construída pela própria instituição, é um instrumento de auxílio, sendo uma alternativa para que o profissional faça sua escolha. Aqui se frisa a importância da criação de remissivas de nomes de autores, a qual contribui tanto nas buscas do usuário como nas do profissional.

O formato MARC 21 e o AACR2, como instrumentos documentários para criação de catálogos, e política institucional para tratamento da informação, cumprem suas funções no estabelecimento destes procedimentos. A escolha e a forma dos pontos de acesso para a recuperação da informação é determinada previamente, mas a decisão sobre como fazer isso a cada registro depende do profissional.

A utilização de um código, no caso, o AACR2, sempre poderá originar mais de uma interpretação. Mesmo com esta constatação, o resultado final registrado no catálogo não deveria apresentar tantas divergências como as observadas em se tratando dos mesmos documentos e autores. No caso analisado, faz-se necessário verificar se a política de manutenção da consistência dos dados descritivos dos registros foi devidamente implementada, ou seja, se os profissionais foram capacitados e sensibilizados para corrigir registros antigos com erro ou para encaminhar essa informação para os responsáveis por esta correção. É necessário verificar também se os critérios para entrada de dados descritivos foram devidamente institucionalizados, no que se refere à definição de itens orientados segundo o AACR2 ou baseados em critérios locais, estabelecidos em manual da instituição.

O profissional deve saber utilizar os instrumentos de organização da informação (no caso estudado, o formato MARC 21 e o AACR2) e discernir sobre a forma como os mesmos são institucionalizados. Não deve abordar estes instrumentos como a causa de todos os problemas, mas saber interpretá-los e empregá-los, propondo soluções que decorram em processos satisfatórios de recuperação da informação. 


\section{REFERÊNCIAS}

ARENDT, Carmen Vera. Sistema de información bibliográfica de la CEPAL: manual de referencia. Santiago de Chile: CEPAL, 2003. (Manuales, 26). Disponível em:

<http://www.eclac.cl/publicaciones/SecretariaEjecutiva/3/LCL1963PE/lcl1963e.pdf>. Acesso em: 12 fev. 2009.

BALBY, Claudia Negrão. Formatos de intercâmbio de registros bibliográficos: conceitos básicos. Cadernos da FFC, Marília, v. 4, n. 1, 1995.

BARBOSA, Alice Príncipe. Novos rumos da catalogação. Rio de Janeiro: BNG/Brasilart, 1978.

BIREME. Manual de descrição bibliográfica - LILACS. 7. ed. São Paulo, 2008. Disponível em: <http://bvsmodelo.bvsalud.org/download/lilacs/LILACS-2-ManualDescricao-pt.pdf>. Acesso em: 10 fev. 2008.

BYRNE, Deborah J. Manual de MARC: cómo interpretar y usar registros MARC. Buenos Aires: Grebyd, 2001.

CORDEIRO, Maria Inês. The UNIMARC landscape: updated overview. Evolving bibliographic standards: the role and place of UNIMARC. Quebec, 2008. (Apresentado na Reunião da IFLA, de 2008, em Quebec).

CYSNEIROS, Luiz Fernando. Formato de comunicação: é necessário? Revista de Biblioteconomia, Brasília, v. 15, n. 2, p. 327-329, jul./dez. 1987.

FERREIRA, Margarida M. MARC 21: formato condensado para dados bibliográficos. 2 . ed. Marília: UNESP, 2002.

FURRIE, Betty. O MARC bibliográfico: um guia introdutório: catalogação legível por computador. Brasília: Thesaurus, 2000.

GARRIDO ARILLA, María Rosa. Teoría e historia de la catalogación de documentos. Madrid: Editorial Síntesis, 1996.

HÜBNER, Edwin. Catálogo coletivo Bibliodata: um produto brasileiro para as bibliotecas brasileiras. In: CONGRESSO INTERNACIONAL DE ARQUIVOS, BIBLIOTECAS, CENTROS DE DOCUMENTAÇÃO E MUSEUS, 1., 2002, São Paulo. Anais ... São Paulo: Imprensa Oficial do Estado, 2002. p. 215-223.

LAFUENTE LÓPEZ, Ramiro; GARDUÑO VERA, Roberto. Lenguajes de marcado de documentos digitales de caracter bibliográfico. México: UNAM, 2001.

LIBRARY OF CONGRESS. The MARC 21 formats: backgrounds and principles. [Washington]: Library of Congress, 1996. Disponível em:

<http://www.loc.gov/marc/96principl.html>. Acesso em: 9 jul. 2007.

. Understanding MARC authority records. [Washington]: Library of Congress, 2004. Disponível em: <http://www.loc.gov/marc/uma/index.html>. Acesso em: 26 jun. 2007.

LOBO, Maria de Fátima Diniz. Intercâmbio de registros entre os formatos bibliográficos IBICT e AGRIS: um estudo comparado. 1990. 158 f. Dissertação (Mestrado em Biblioteconomia e Documentação) - Faculdade de Estudos Sociais e Aplicados, Universidade de Brasília, Brasília, 1990. 
LÓPEZ YEPES, José (Ed.). Diccionario enciclopédico de ciencias de la documentación. Madrid: Editorial Síntesis, 2004. 2v.

MARCONDES, Carlos Henrique ; SAYÃO, Luiz Fernando. Situação brasileira dos formatos de intercâmbio bibliográfico e dos "software" de suporte. In: SEMINÁRIO NACIONAL DE BIBLIOTECAS UNIVERSITÁRIAS, 7., 1991, Rio de Janeiro. Anais ... Rio de Janeiro, 1991. p. 241-255.

MARTIN, Philippe. Format. In: CACALY, Serge (Coord.). Dictionnaire encyclopédique de l'information et de la documentation. Paris: Nathan, 1997.

MELGAÇO, Leda Maria Louzada. Formato IBICT: formato de intercâmbio bibliográfico e catalográfico. In: CONGRESSO BRASILEIRO DE BIBLIOTECONOMIA E DOCUMENTAÇÃO, 14., 1987, Recife. Anais... Recife: Associação Profissional de Biblioteconomia de Pernambuco, 1987. p. 139-151.

MEY, Eliane Serrão Alves. Considerações (preguiçosas) sobre a prática da catalogação. Revista de Biblioteconomia de Brasília, v.19, n.2, p. 127-136, jul./dez. 1995a. Introdução à catalogação. Brasília: Briquet de Lemos, 1995b.

NOGUEIRA, Adriana Hypólito; SALEM, Aziz Donizetti Cavalheiro. Banco de dados bibliográficos da USP-DEDALUS: uma proposta de atualização. São Paulo: [S.n.], 2001.

ORTEGA, Cristina Dotta. Introdução ao microISIS. 2. ed. Brasília: Briquet de Lemos, 2002.

ORTIZ-REPISO JIMÉNEZ, Virginia. Análisis documental formal: la catalogación. In: LÓPEZ YEPES, José (Coord.). Manual de ciencias de la documentación. 2. ed. Madrid: Ediciones Pirámide, 2006. p. 359-377.

RíOS HILÁRIO, Ana Belén. Nuevos horizontes en el análisis de los registros y la normativa bibliográfica. Gijón: Ediciones Trea, 2003.

ROBREDO, Jaime. Documentação de hoje e de amanhã: uma abordagem revisitada e contemporânea da Ciência da Informação e de suas aplicações biblioteconômicas, documentárias, arquivísticas e museológicas. 4. ed. Brasília: Edição de autor, 2005.

UNIVERSIDADE DE SÃO PAULO. Banco de dados bibliográficos da USP: catálogo online global - DEDALUS. São Paulo. Disponível em:

<http://dedalus.usp.br:4500/ALEPH/POR/USP/USP/USP. Acesso: 15 dez. 2008.

SISTEMA INTEGRADO DE BIBLIOTECAS. Bibliotecas. São Paulo, 2001. Disponível em: <http://www.usp.br/sibi/>. Acesso em: 8 nov. 2007.

São Paulo, 2001-2009. SIBinet. Sobre o SIBi/USP. Regulamentação. Portaria GR-2922. Disponível em: <http://www.usp.br/sibi/>. Acesso em: 01 de jun 2009.

SVENONIUS, Elaine (Ed.). The conceptual foundations of descritive cataloging. San Diego: Academic Press, 1989.

WINKE, R. Conrad. Discarding the main entry in an online cataloging environment. Cataloging \& Classification Quartely, v.16, n. 1, p. 53-70, 1993. 


\title{
Title
}

An analysis of information retrieval in university library's on-line catalog

\begin{abstract}
This work aims to analyze the information retrieval in on-line catalogs of university libraries through the observation of data input of the MARC 21 format. The methodology includes conceptual and literature review that characterizes and presents cataloging rules and bibliographical records formats as well as other variables related to information retrieval. It also includes the analysis of two searches conducted in the main catalog of the Sao Paulo's University catalog. This analysis demonstrates that a simple task, as the search in the catalogs, may present some problems for users. It concludes that the librarians and other library assistants involved in cataloging practices must have control over the stages and tools for information processing, as this process involves investigative and interpretative capacity, and also decision-making.
\end{abstract}

\section{Keywords}

On-line catalog; University libraries; Information retrieval; MARC format; AACR2

\section{Title}

Análisis de la recuperación de la información en catálogo en línea de biblioteca universitaria

\section{Resumen}

El trabajo pretende analizar la recuperación de la información en un catálogo en línea de biblioteca universitária, por medio de la observación del ingreso de datos en formato MARC 21. La metodología incluye revisión bibliográfica que conceptúa y caracteriza reglas de catalogación y formatos de registro bibliográfico, presentando el papel de ambos y de otras variables en los resultados de la recuperación de la información. Incluye también el análisis de los resultados de dos búsquedas realizadas en el catálogo general de las bibliotecas de la Universidade de São Paulo. Este análisis demuestra que una tarea que, parece sencilla como la búsqueda en catálogos, presenta problemas a los usuarios. Además, se concluye que los bibliotecarios y auxiliares involucrados en la práctica de la catalogación deben poseer dominio sobre los procesos e instrumentos del tratamiento de la información de la unidad de trabajo en la que actúan. Tal dominio se implica en capacidad investigadora, interpretativa y toma de decisión.

\section{Palabras clave}

Catalogo en línea; Bibliotecas universitarias; Recuperación de la información; Formato MARC; AACR2

Recebido em: 06.01.2009

Aceito em: 30.05.2009 